\title{
Rediagnosis of Lung Cancer as NUT Midline Carcinoma Based on Clues From Tumor Genomic Profiling
}

\author{
Alexander S. Baras, MD, PhD; ; Jarushka Naidoo, $\mathrm{MBBCh}^{\mathrm{a}}$; Christine L. Hann, MD, PhD; \\ Peter B. Illei, MD; ${ }^{\mathrm{a}}$ Charles W. Reninger III, $\mathrm{MD}^{\mathrm{b}}$; and Josh Lauring, MD, PhD
}

\begin{abstract}
Tumor DNA sequencing can identify rare driver genomic alterations that suggest targets for cancer therapy, even when these drivers cannot be suspected on clinical grounds. In some cases, genomic alterations identified in the tumor can lead to a change in diagnosis with implications for prognosis and therapy. This report describes a case in which evaluation of tumor sequencing results by a molecular tumor board (MTB) led to rediagnosis of a non-small cell lung cancer as highly aggressive NUT midline carcinoma, with implications for targeted therapy using an investigational bromodomain and extraterminal (BET) inhibitor. We discuss the molecular biology and diagnosis of this rare tumor, and suggest how improved annotation of tumor sequencing reports and multidisciplinary expertise of MTBs can facilitate timely diagnosis of rare tumors and application of potential targeted therapies.
\end{abstract}

J Natl Compr Canc Netw 2018;16(5):467-472 doi: 10.6004/jnccn.2017.7203

\section{Case Report}

A 39-year-old man who never smoked and had no significant past medical history presented to an urgent care clinic with a productive cough. A chest radiograph described an ill-defined density at the left hilum. He was treated with azithromycin and advised to follow up within 10 days to reevaluate the abnormal radiographic findings. A repeat chest radiograph showed the same findings suspicious for malignancy, and he was referred for chest CT, which demonstrated a left lower lobe mass concerning for lung cancer or lymphoma (Figure 1A). PET/CT subsequently showed a left lower lobe lung mass measuring 12 x $6 \times 7 \mathrm{~cm}$ with intense metabolic activity invading the hilum, bulky mediastinal adenopathy, and another left lower lobe cavitary mass. Multiple lytic bone lesions were

\footnotetext{
aSidney Kimmel Comprehensive Cancer Center at Johns Hopkins, Baltimore, Maryland, and 'Hematology and Oncology Consultants of Pennsylvania, Camp Hill, Pennsylvania.

Submitted October 12, 2017; accepted for publication December 21, 2017.

Dr. Hann has disclosed that she has received research funding from GlaxoSmithKline, AbbVie, Bristol-Myers Squibb, and Merrimack Pharmaceuticals; and is a consultant for AbbVie, Bristol-Myers Squibb, and Genentech. Dr. Illei has disclosed that he is on an advisory board or is a consultant for AstraZeneca, Bristol Myers Squibb, Roche, and Abbvie. Dr. Naidoo has disclosed that she has received research funding from Merck, AstraZeneca/Medimmune, and Kyowa Kirin; is a consultant for Bristol Myers Squibb, AstraZeneca/Medimmune, and Takeda; and
}

identified in the spine, pelvis, and femur (Figure 1B). A brain MRI was negative for metastases. Laboratory tests were notable only for minimally elevated aspartate aminotransferase (39 U/L) and alanine aminotransferase (45 U/L). Fiberoptic bronchoscopy was performed with fineneedle aspiration (FNA) of a station 7 lymph node, and the pathology was read as poorly differentiated squamous cell carcinoma (SCC) (Figure 2). Immunostains were positive for p63 and negative for TTF-1, napsin A, and synaptophysin. Tumor cells were negative for PD-L1, and tumor-associated immune cells showed 5\% staining (antibody SP142). Tissue was sent for sequencing of a 315 cancer-related gene panel (FoundationOne, Foundation Medicine). By the time the patient saw an oncologist, he had lost 10 to 15 pounds. He was referred for consultation at Johns Hopkins University, where repeat PD-L1

has received honoraria from Bristol Myers Squibb and AstraZeneca/ Medimmune. Dr. Lauring has disclosed that he is a consultant for the Jackson Laboratory. The remaining authors have disclosed that they have no financial interests, arrangements, affiliations, or commercial interests with the manufacturers of any products discussed in this article or their competitors.

This work was supported in part by NIH P30 CA006973.

Correspondence: Josh Lauring, MD, PhD, Sidney Kimmel Comprehensive Cancer Center at Johns Hopkins, Cancer Research Building 1, Room 146, 1650 Orleans Street, Baltimore, MD 21287.

Email: jlauring@jhmi.edu 
staining with the 22C3 antibody demonstrated a tumor proportion score of $1 \%$ to $2 \%$ (low). Several immunotherapy clinical trials were discussed, as well as standard platinum-doublet chemotherapy.

The patient's tumor sequencing test and clinical history were also referred to the multidisciplinary GAITWAY molecular tumor board (MTB) at Johns Hopkins University to review potentially actionable genomic findings. ${ }^{1}$ Only 2 alterations were described in the report: a bromodomain-containing 4 (BRD4)
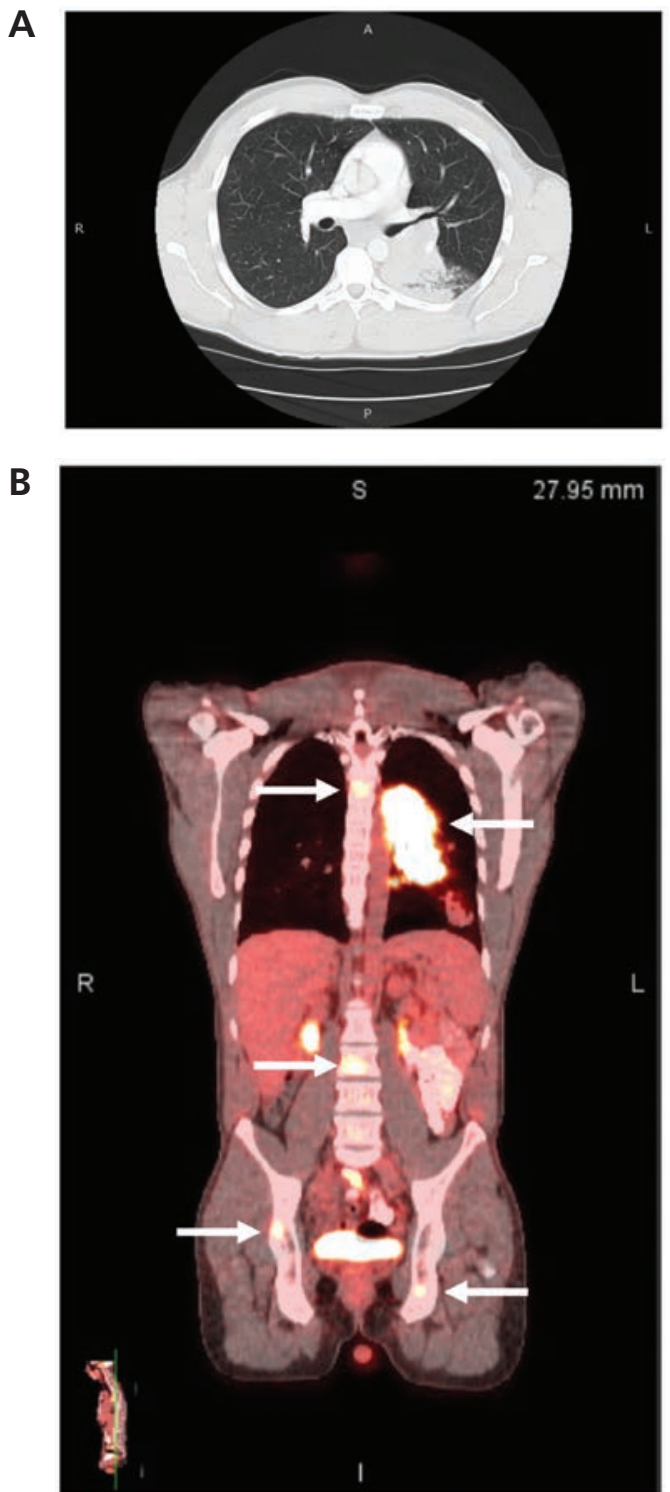

Figure 1. Diagnostic imaging. (A) Cross-sectional CT image shows a left lower lobe lung mass with associated postobstructive changes. (B) Fused coronal PET/CT image shows an intensely FDG-avid left lower lobe lung mass and confluent mediastinal lymphadenopathy, as well as FDG-avid lesions in the thoracic and lumbar spine and pelvis (arrows).
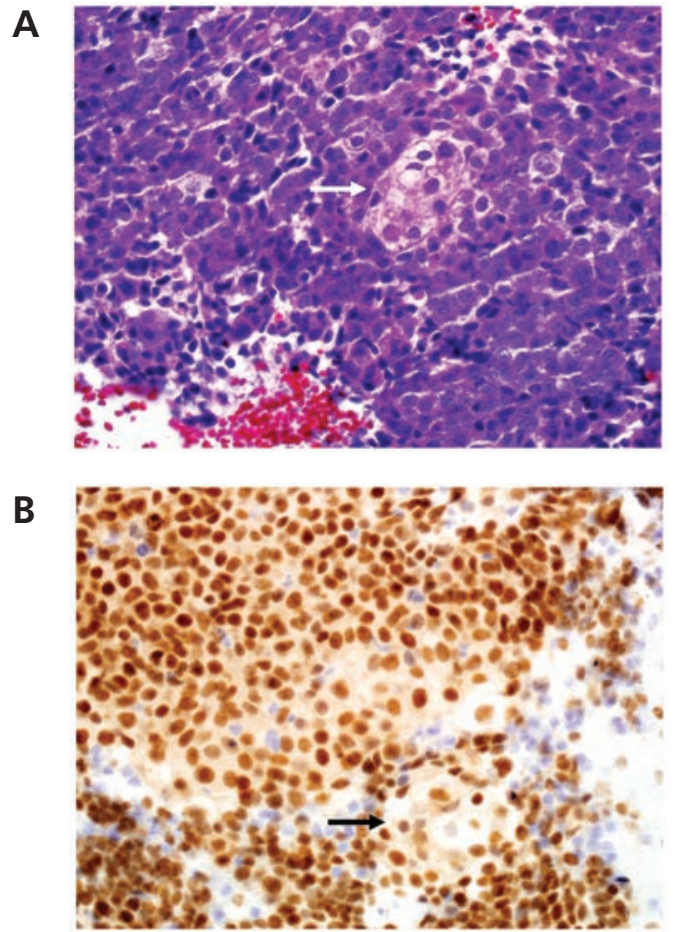

Figure 2. Pathologic characterization of tumor (original magnification $x 200$ ). (A) Hematoxylin and eosin stain of a cell block from a station 7 lymph node fine-needle aspirate shows a poorly differentiated epithelial neoplasm with focal areas of abrupt squamous differentiation (white arrow). (B) Immunohistochemical staining for NUT protein (anti body C52B1, 1:50 dilution, Cell Signaling Technology) shows strong, diffuse, nuclear staining throughout the tumor. Black arrow indicates focal area of abrupt squamous differentiation.

rearrangement in exon 11 and a MutL homolog 1 (MLH1) R325Q mutation. The report stated that the test's sensitivity was limited by sample quality. Microsatellite stability status was reported as unknown and tumor mutation burden was not calculated, also presumably because of inadequate sample quality or DNA quantity. The MLH1 R325Q mutation has been reported previously in the germline in a few Lynch syndrome families, but it has not been associated with microsatellite instability, and in vitro functional assays show that it is mismatch repair-proficient. ${ }^{2}$ This variant has been classified as likely benign. ${ }^{3}$ The patient's family history was not suggestive of Lynch syndrome, with a paternal grandfather with lung cancer (heavy smoker) and a paternal grandmother with a gynecologic cancer (age unknown). The MTB did not recommend germline testing or further workup.

The tumor sequencing report stated that alterations removing the $\mathrm{C}$ terminus of BRD4 are predicted to disrupt its transcriptional and chromatin regula- 
tory functions, although this particular alteration was insufficiently characterized and its effect on protein function was unclear. No off-label therapies or clinical trials were listed on the report for the BRD4 alteration.

The MTB observed that BRD4 rearrangements have been reported to occur in NUT midline carcinoma (NMC), and that reported breakpoints in several cases involved exon 11 of BRD4. NMC is a rare entity, although its true prevalence is unknown. It was originally described as a highly aggressive form of poorly differentiated carcinoma with variable degrees of squamous differentiation, occurring in the midline in young patients. ${ }^{4,5}$ Molecular genetic studies have demonstrated recurrent fusions involving the NUT gene (NUT family member 1 , also known as NUTM1) on chromosome $15 \mathrm{q} 14$. Most reported fusions involve BRD4 on chromosome 19p13.1; other cases with NUT fused to the related bromodomain protein BRD3 or the histone methyltransferase NSD3 have been reported. ${ }^{6,7}$ More recent studies have identified the pathognomonic rearrangements in patients ranging in age from 0.1 to 81 years, with a median age of 29 years. ${ }^{8}$

$\mathrm{NMC}$ is a very aggressive cancer with a median survival of only 6.7 months in the largest case series reported. ${ }^{9}$ Survival for adult patients at 1 year is $<20 \%$. There is no accepted standard of care, but the best reported outcomes have been in patients with primary head and neck tumor sites treated with intensive local therapy, including gross total resection and/or radiation or chemoradiation. ${ }^{8,9}$ Chemotherapy regimens for SCC, sarcomas, and germ cell tumors have been used and frequently include platinum drugs, anthracyclines, and alkylating agents, but no regimen has shown clear superiority. Initial responses to chemotherapy have been reported, but the tumor typically recurs quickly as a refractory, rapidly progressive cancer. ${ }^{9,10}$ Only a few long-term survivors have been reported, all of whom received intensive combination chemotherapy and radiation. ${ }^{10-12}$ Several of these patients were treated according to the Scandinavian Sarcoma Group IX protocol using alternating courses of vincristine, doxorubicin, and ifosfamide with cisplatin, doxorubicin, and ifosfamide. ${ }^{11,12}$

Preclinical functional studies have demonstrated that the BRD4-NUT fusion results in extensive recruitment of NUT to large "megadomains" of DNA ranging from hundreds of kilobases to megabases, through tethering by the acetyl-histone-binding BET (bromodomain and extraterminal) domain of BRD4. Increased histone lysine acetylation leads to feed-forward recruitment of more BRD4 to these loci, causing widespread epigenetic transcriptional activation, including key loci such as MYC. ${ }^{13}$ Recently, several pharmaceutical companies have developed inhibitors of BET domain-containing proteins, including BRD4. These drugs are being tested in ongoing clinical trials, some of which have cohorts for NMC. A recent case series described dramatic, rapid responses of several cases of NMC to one of these investigational BET inhibitors, OTX015/MK-8628.14 Of 10 evaluable patients with NMC, 2 responses and 4 cases of stable disease were reported in a phase I/ II trial of the BET inhibitor GSK525762. ${ }^{15}$ There is also a case report of a response to the histone deacetylase inhibitor vorinostat. ${ }^{16}$

The MTB observed that the patient was relatively young at 39 years of age and was a never-smoker, despite having been diagnosed with SCC of the lung. Given the histology and the BRD4 rearrangement, the MTB was suspicious that his disease was in fact NMC, and suggested performing immunohistochemistry for the NUT protein, which is highly sensitive and specific for NMC. ${ }^{17}$ After some delay in obtaining remaining tissue from the patient's FNA, the specimen tested positive for NMC with strong, diffuse NUT staining (Figure 2).

In the interim, the patient experienced worsening bone pain, dyspnea, and fever, and was hospitalized locally. CT imaging demonstrated an increase in the primary lung mass and mediastinal adenopathy, with encasement of the left airways and postobstructive changes, new liver lesions, and T5 vertebral compression fracture. Hepatic transaminases were both $>1.5$ times the upper limit of normal. Given the constraints of his worsening performance status, he was treated with radiation to the mediastinum and lung mass with concurrent weekly dosing of carboplatin and paclitaxel (subsequently changed to nab-paclitaxel). He was discharged and received further radiotherapy to the right hip and sacrum, kyphoplasty, and denosumab. His symptoms improved and he was seen again at Johns Hopkins University nearly 3 months after his tumor sequencing report was issued to discuss participation in a clinical trial of the BET inhibitor GSK525762 with a cohort for NMC (ClinicalTrials. gov identifier: NCT01587703). Unfortunately, he 
Baras et al

had abnormal hepatic transaminase levels that precluded immediate trial participation, and several days later was readmitted to the hospital with epidural spinal cord compression at T2. He underwent surgical decompression and then initiated treatment with doxorubicin and cyclophosphamide. However, he had continued progression of bone metastases and died before the second cycle of chemotherapy.

Analysis of the raw sequencing data from the patient's tumor sequencing test performed after his death revealed a complex set of chromosomal rearrangements involving the BRD4 locus on chromosome 19p13.1, with multiple breakpoints in which a portion of chromosome 6 is inserted into the intronic sequence downstream of exon 11 in BRD4, with respect to the transcriptional orientation (Figure 3). This is then followed by an apparent inversion of a short subsequent chromosome 19 segment, which then harbors a breakpoint to chromosome 15 upstream of the NUTM1 locus. There is also evidence of a breakpoint downstream to these events, with respect to the BRD4 locus, involving chromosome 8 . There was no definitive evidence of a rearrangement locating the upstream BRD4 exons (1-11) into the NUTM1 locus in the same transcriptional orientation. Given the targeted nature of this sequencing assay, which does not directly have sequencing baits/probes for NUTM1, we were unable to definitively evaluate whether additional breakpoints within this region might be present that would support a BRD4-NUTM1 fusion. However, the combination of the various genomic alterations in the BRD4 locus (including a breakpoint to just upstream of the NUTM1 locus) and the strong, diffuse NUT immunohistochemical staining is suggestive of a cryptic BRD4-NUT fusion or some form of transcriptional upregulation of NUT as a consequence of the rearrangement.

\section{Discussion}

This case illustrates a missed opportunity to match a promising targeted therapy with a specific genomic driver alteration in a rare cancer type. Many oncologists may not be aware of NMC as a clinical entity. Although outcomes to date have been poor, making the correct diagnosis can lead to early use of aggressive combined modality therapy, which offers the best chance for disease control. Specific diagnosis



Figure 3. Complex chromosomal rearrangements within the BRD4 locus involving chromosomes $6,8,15$, and 19 . (A) The DNA sequence read alignments at the BRD4 locus are shown, with the chromosomal region and BRD4 exon structure indicated at the top of the figure. Reads are organized by chromosome of origin of the mate pair. As expected, most reads have both mates mapping to the $B R D 4$ locus on chromosome 19 (only a portion of these reads are shown in the figure). A smaller number of reads have one mate pair mapping to BRD4 on chromosome 19 and the other mate pair mapping to chromosome 6, 8, or 15 (red box), indicating a complex pattern of chromosomal rearrangements clustered distal to BRD4 exon 11. (B) The DNA sequence read alignments near the NUTM1 locus are shown, in which the chromosome 19 to 15 rearrangement is present upstream of the NUTM1 locus (red box). All reads are stratified by the chromosome of the mate pair and overlaid by the labeled gene structures. 
also allows for enrollment of patients with NMC on clinical trials, which may define the role of novel agents in the management of this disease. Evidence for BET inhibitors in this disease is still preliminary, and we do not know whether this patient would have responded, but this approach represents the kind of specific therapeutic targeting that tumor sequencing is intended to enable. The MTB's ability to discern the possibility of NMC based on the reported BRD4 rearrangement, together with the clinical and pathology data, illustrates one of the many ways MTBs can add value in interpreting whether tumor molecular profiling results are clinically actionable.

It is likely that other cases of NMC are diagnosed as poorly differentiated SCC of the lung or other sites, although it will be difficult to define how often this occurs until more widespread molecular testing of the relevant genes is reported. One large series of unselected lung carcinomas examined for NUT expression did not identify any positive cases. ${ }^{18}$ However, over a 4-year period, a specialty referral center described 8 cases of primary pulmonary NMC, which shared very similar clinical features to this case. ${ }^{19}$ Retrospective analysis by this group of 166 consecutive lung cancer biopsies lacking glandular differentiation identified 1 case as NMC (0.6\%). Clinicians should consider NMC in the differential diagnosis of poorly differentiated carcinomas occurring in the head and neck or thorax, particularly in nonsmokers.

This tumor had a complex set of chromosomal rearrangements, and the genomic coverage of this test does not conclusively link the upstream exons 1-11 of BRD4 to NUTM1. The cautious language of the report is certainly accurate and appropriate. DNA-based cancer gene panel tests have limitations in their ability to identify gene fusions, and sensitivity is highest when specific baits are designed to capture the exon or intron sequences expected to be involved. Despite the sequencing test's inability to identify the canonical BRD4-NUT rearrangement in this case, the clinical presentation, histopathology, and NUT immunohistochemistry all support the diagnosis of NMC. It is possible that NUT overexpression is activated in this case by disruption of transcriptional regulatory elements near the NUTM1 locus, rather than by a gene fusion. However, we favor the existence of a cryptic rearrangement, given that the BRD4 locus rearrangement is probably not coincidental. Of note, complex multichromosome rearrangements due to chromoplexy that lead to productive BRD4-NUT fusion transcripts have been identified using whole-exome sequencing in NMC. ${ }^{20}$

Even in the absence of definitive evidence of a typical BRD4-NUT fusion in this case, an annotation that had commented on the existence of BRD4NUT rearrangements in NMC and the possibility of confirmatory testing in the appropriate clinical context (poorly differentiated SCC) could have directed a clinician toward the appropriate diagnostic workup and clinical trial options for NMC. As noted earlier, NUT immunohistochemistry is a reliable and simple method to identify NMC. Other methods include cytogenetics, fluorescence in situ hybridization, and RNA-based approaches, such as next-generation RNA sequencing, RT-PCR, and the Archer FusionPlex assay (ArcherDX, Inc.). Tumor sequencing providers currently suggest considering additional testing in other situations. For example, annotations for deleterious mutations in genes such as BRCA1 and BRCA2 advise that in some cases these could represent germline cancer-predisposing variants and suggest germline testing if clinically indicated.

\section{Conclusions}

This case highlights a disconnect between traditional pathology and commercial tumor gene sequencing, wherein sequencing results are often provided back to oncologists or surgeons and may not be reviewed by the pathologists who originally reviewed the tissue, missing the opportunity to revise a diagnosis accordingly. Rare tumors will always pose clinical challenges, but improvements to tumor sequencing annotation and more accessible and timely review of findings by MTBs with both oncology and molecular pathology expertise could refine clinical diagnoses and match patients, such as the one discussed herein, to appropriate molecularly targeted clinical trials before the therapeutic window closes.

\section{Acknowledgments}

The authors would like to thank the entire GAITWAY MTB for their input and assistance with this case. 
Baras et al

\section{References}

1. Dalton WB, Forde PM, Kang $\mathrm{H}$, et al. Personalized medicine in the oncology clinic: implementation and outcomes of the Johns Hopkins molecular tumor board. JCO Precis Oncol 2017:1-19.

2. Borras $E$, Pineda $M$, Brieger $A$, et al. Comprehensive functional assessment of MLH1 variants of unknown significance. Hum Mutat 2012;33:15761588 .

3. Thompson BA, Spurdle AB, Plazzer JP, et al. Application of a 5-tiered scheme for standardized classification of 2,360 unique mismatch repair gene variants in the InSiGHT locus-specific database. Nat Genet 2014:46:107-115.

4. French CA, Kutok JL, Faquin WC, et al. Midline carcinoma of children and young adults with NUT rearrangement. J Clin Oncol 2004:22:41354139.

5. French CA, Miyoshi I, Kubonishi I, et al. BRD4-NUT fusion oncogene: a novel mechanism in aggressive carcinoma. Cancer Res 2003;63:304-307.

6. French CA, Rahman S, Walsh EM, et al. NSD3-NUT fusion oncoprotein in NUT midline carcinoma: implications for a novel oncogenic mechanism. Cancer Discov 2014;4:928-941.

7. French CA, Ramirez CL, Kolmakova J, et al. BRD-NUT oncoproteins: a family of closely related nuclear proteins that block epithelial differentiation and maintain the growth of carcinoma cells. Oncogene 2008;27:2237-2242.

8. Chau NG, Hurwitz S, Mitchell CM, et al. Intensive treatment and survival outcomes in NUT midline carcinoma of the head and neck. Cancer 2016;122:3632-3640.

9. Bauer DE, Mitchell CM, Strait KM, et al. Clinicopathologic features and long-term outcomes of NUT midline carcinoma. Clin Cancer Res 2012;18:5773-5779.

10. Lemelle L, Pierron G, Freneaux P, et al. NUT carcinoma in children and adults: a multicenter retrospective study [published online June 23, 2017]. Pediatr Blood Cancer. doi: 10.1002/pbc.26693
11. Mertens F, Wiebe T, Adlercreutz $\mathrm{C}$, et al. Successful treatment of a child with t(15;19)-positive tumor. Pediatr Blood Cancer 2007;49:1015-1017.

12. Storck S, Kennedy AL, Marcus KJ, et al. Pediatric NUT-midline carcinoma: therapeutic success employing a sarcoma based multimodal approach. Pediatr Hematol Oncol 2017;34:231-237.

13. Alekseyenko AA, Walsh EM, Wang X, et al. The oncogenic BRD4-NUT chromatin regulator drives aberrant transcription within large topological domains. Genes Dev 2015;29:1507-1523.

14. Stathis A, Zucca E, Bekradda M, et al. Clinical response of carcinoma harboring the BRD4-NUT oncoprotein to the targeted bromodomain inhibitor OTX015/MK-8628. Cancer Discov 2016;6:492-500.

15. O'Dwyer PJ, Piha-Paul SA, French C, et al. GSK525762, a selective bromodomain (BRD) and extra terminal protein (BET) inhibitor: results from part 1 of a phase I/II open-label single-agent study in patients with NUT midline carcinoma (NMC) and other cancers [abstract]. Cancer Res 2016;76(14 Suppl):Abstract CT014.

16. Maher OM, Christensen AM, Yedururi S, et al. Histone deacetylase inhibitor for NUT midline carcinoma. Pediatr Blood Cancer 2015;62:715 717.

17. Haack H, Johnson LA, Fry CJ, et al. Diagnosis of NUT midline carcinoma using a NUT-specific monoclonal antibody. Am J Surg Pathol 2009;33:984-991

18. Lund-Iversen M, Groholt KK, Helland A, et al. NUT expression in primary lung tumours. Diagn Pathol 2015;10:156.

19. Sholl LM, Nishino M, Pokharel S, et al. Primary pulmonary NUT midline carcinoma: clinical, radiographic, and pathologic characterizations. J Thorac Oncol 2015;10:951-959.

20. Lee JK, Louzada S, An Y, et al. Complex chromosomal rearrangements by single catastrophic pathogenesis in NUT midline carcinoma. Ann Oncol 2017;28:890-897.

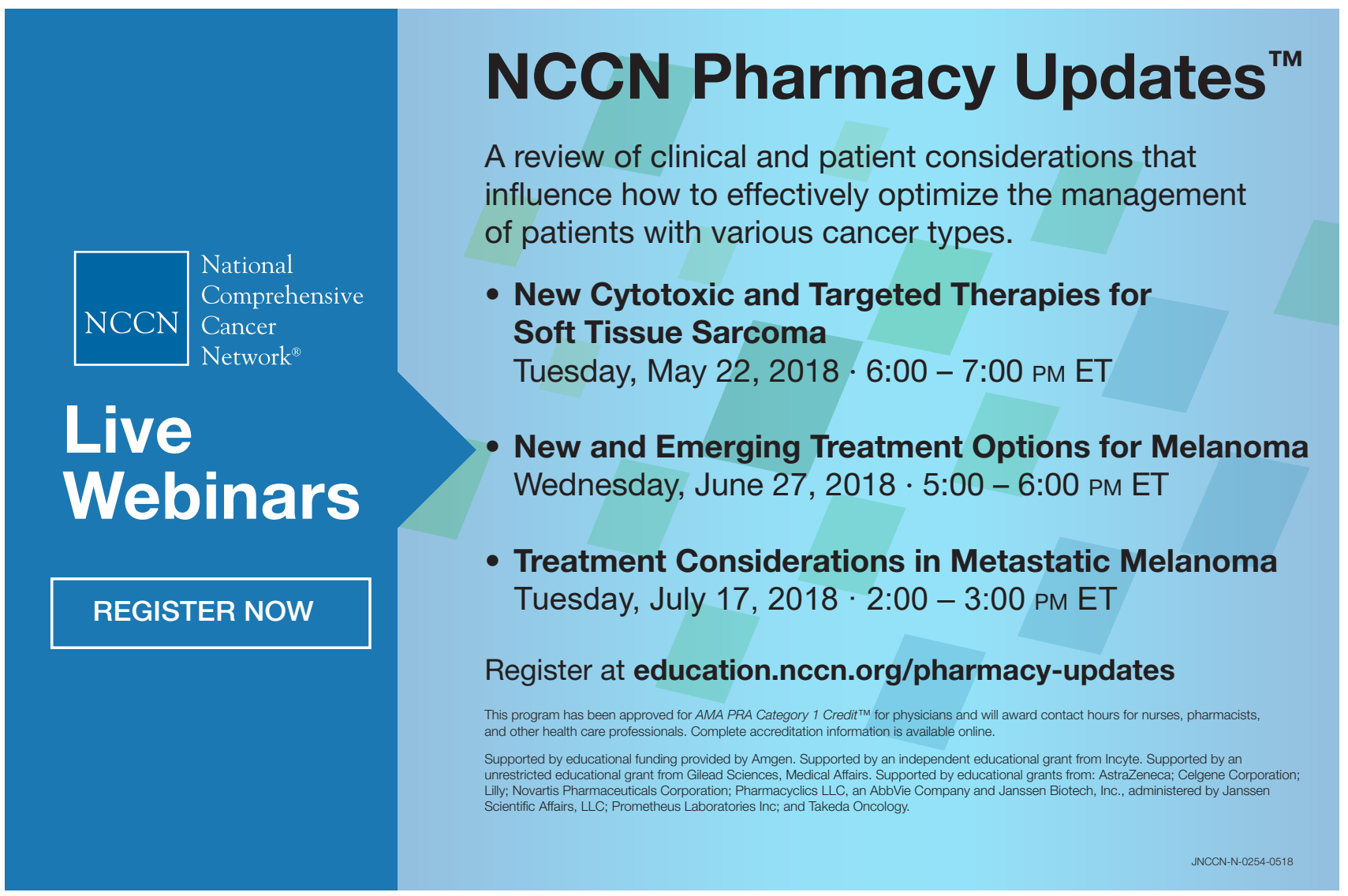

